Proc. of the 11 Int. School on Theoretical Physics Symmetry and Structural Properties of Condensed Matter, Rzeszów 2014

\title{
Effect of Majorana Fermions on Andreev Spectroscopy of Multiband Topological Superconductors
}

\author{
A.C. Silva ${ }^{a}$, M.A.N. ARAÚJO ${ }^{a, b, c, *}$ AND P.D. SACRAMENTO ${ }^{a, c}$ \\ ${ }^{a}$ CFIF, Instituto Superior Técnico, Universidade de Lisboa, Av. Rovisco Pais, 1049-001 Lisboa, Portugal \\ ${ }^{b}$ Departamento de Física, Universidade de Évora, P-7000-671, Évora, Portugal \\ ${ }^{c}$ Beijing Computational Science Research Center, Beijing 100089, China

\begin{abstract}
We introduce a model for a multiband topological superconductor with two orbitals per lattice site, in two spatial dimensions. Concentrating on the Andreev reflection problem, the appropriate wave function matching conditions for an interface with a normal single-band metal were previously derived in the framework of a quantum waveguide theory. This theory retrieves the correct number of Majorana fermion states as predicted by the topological index. We obtain the differential conductance as a function of bias voltage, which displays the contribution of the Majorana fermions. Interface disorder is also considered. By varying band structure parameters, topological transitions can be induced, whereby the number of the Majorana modes varies. We calculate the effect of such transitions on the differential conductance.
\end{abstract}

DOI: 10.12693/APhysPolA.128.210

PACS: 71.10.Fd, 71.10.Pm, 71.10.Li, 73.20.-r, 74.45.+c, 74.20.Rp, 74.70.-b

\section{Introduction}

Recent interest in non-trivial topological properties of insulators [1] and superconductors [2,3] has spurred intensive research on band models displaying non-trivial topology [4].

We introduce below a model for a superconductor which has two orbitals per lattice site but no spinorbit coupling and study the Andreev problem for a normal metal/superconductor boundary $(\mathrm{N} / \mathrm{S})$. Assuming the normal metal to be single band, the previously established quantum waveguide theory (QWT) $[5,6]$ for the wave function matching conditions at the interface is used. While the Blonder-Tinkham-Klapwijk (BTK) theory [7] predicts a zero energy Andreev bound state (ABS), which is a Majorana fermion (MF) in this case, for each electron pocket, we show that QWT accounts for the correct number of MF's expected from the topological index. The purpose of this work is therefore twofold: (i) as we shall see below, the ABS predicted by QWT correctly reconciles the Andreev problem with the topological index; (ii) we wish to see the interference effects on the differential conductance, as the incident electrons go from a single band metal to a multiband superconductor (MBS), as described by QWT theory.

\section{Model Hamiltonian}

We now construct a model for a topological MBS, with two orbitals per lattice site (pseudo-spin) of a square lattice in addition to the spin degree of freedom.

We write kinetic energy for $\uparrow$-spin electrons as $\boldsymbol{h}(\boldsymbol{k}) \cdot \boldsymbol{\tau}+$ $h_{0}(\boldsymbol{k}) \tau_{0}$ where the Pauli matrices $\tau_{i=1,2,3}$ act on orbital space (pseudo-spin) and $\tau_{0}$ denotes the identity matrix

\footnotetext{
* corresponding author; e-mail: mana@uevora.pt
}

$$
\begin{aligned}
& h_{x}=\sin k_{y}, \quad h_{y}=-\sin k_{x}, \\
& h_{z}=2 t_{1}\left(\cos k_{x}+\cos k_{y}\right)+4 t_{2} \cos k_{x} \cos k_{y}, \\
& h_{0}=-\mu-t_{1}\left(\cos k_{x}+\cos k_{y}\right) .
\end{aligned}
$$

No spin-orbit coupling exists. We assume pairing with spin- $\downarrow$ electrons, whose kinetic energy is related to that of $\uparrow$-spin electrons by time-reversal. One can prove [8] that the topological properties of the superconductor do not at all depend on the topological properties of the normal bands, and non-trivial topology requires $p$-wave pairing. We consider a triplet pairing with odd parity

$$
\widehat{\Delta}(\boldsymbol{k})=d_{z}(\boldsymbol{k}) \sigma_{x} \otimes \tau_{0},
$$

with $d_{z}(\boldsymbol{k})=\Delta\left(\sin k_{x}-i \sin k_{y}\right)$. The Pauli matrix $\sigma_{x}$ operates in spin space. The Bogolyubovde Gennes (BdG) matrix in the particle-hole basis, $\left(\left(\widehat{c}_{\uparrow}\right)\left(\widehat{c}_{\downarrow}\right)\left(\widehat{c}_{\uparrow}^{\dagger}\right)\left(\widehat{c}_{\downarrow}^{\dagger}\right)\right)$, splits into two $4 \times 4$ matrices.

We consider now only the subspace $\left(\left(\widehat{c}_{\uparrow}\right)\left(\widehat{c}_{\downarrow}^{\dagger}\right)\right)$. The parameter choice $\mu=0.6, t_{1}=0.07, t_{2}=-0.08$, produces a Fermi surface with 3 pockets centered at $(0,0)$, $(0, \pi),(\pi, 0)$ in the Brillouin zone, as Fig. 1 (left part) shows. We calculate the topological index (the Chern number) following a method for multiband systems [9] and obtain $C=+1$, indicating that one MF exists. The energy spectrum for an infinite ribbon in the $y y$ (or $x x$ ) direction, for $\Delta=0.1$, is shown in Fig. 2, where the MF is clearly seen at longitudinal momentum $\pi$. If we consider the Andreev problem for a $\mathrm{N} / \mathrm{S}$ interface along $y y$, the MF is detected when the incident electrons have transverse momentum $k_{y}=\pi$ and thus traverse only one FS pocket, at $(0, \pi)$. By reversing the sign of the hopping parameter $t_{2}$, a topologically trivial phase is obtained, with zero Chern number, and the Fermi surface now contains 4 pockets, as can be seen from the right part of Fig. 1, and no MF's should exist.

In the framework of single band BTK theory, each Fermi pocket should contain one zero energy Andreev 

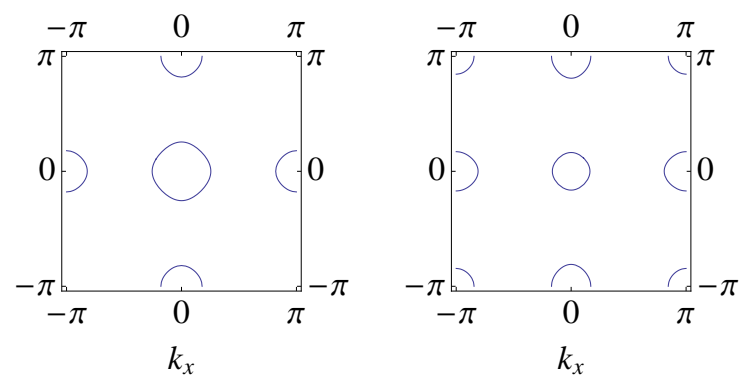

Fig. 1. Fermi surface pockets for model (1)-(2), with $t_{2}=-0.08$ (left) and $t_{2}=0.08$ (right).

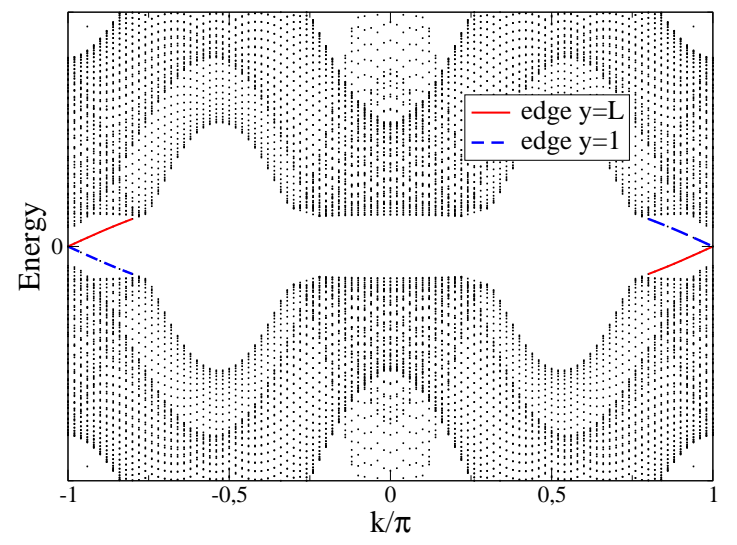

Fig. 2. Spectrum of model (1)-(2) for a ribbon geometry. $t_{2}=-0.08$.

bound state. This is because the pairing function is odd, so that the electrons feel a sign change in the gap function upon specular reflection at the superconductor's surface [10]. Thus 3 or 4 ABS's or MF's are predicted by BTK theory, at conflict with the topological properties. The single MF observed when only a single FS pocket is crossed implies that somehow the two Andreev bound states predicted by BTK theory for the pockets $(0,0)$ and $(\pi, 0)$ should interfere destructively when the incident electron has transverse momentum $k_{y}=0$.

\section{The Andreev problem}

We consider a N/S boundary along $y y$ axis. The incident electron from a single band normal metal will split into the two pseudo-spin channels of the superconductor, as Fig. 3 explains.

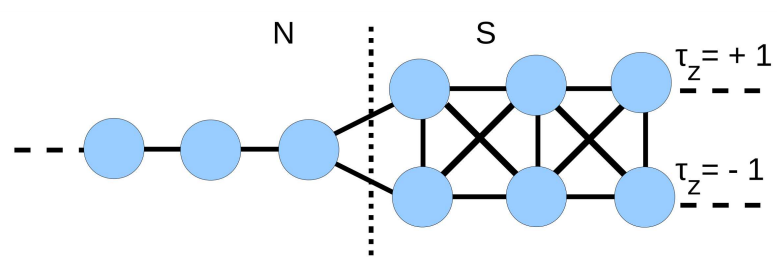

Fig. 3. Tight-binding showing the splitting of the incoming electron into two pseudo-spin channels of the superconductor, similar to a waveguide.
In the $N$ side, $x \leq 0$, the wave function for electrons near the Fermi level is $\exp \left(\mathrm{i} k_{y} y\right) \psi_{N}$ where

$$
\begin{aligned}
& \psi_{N}(x \leq 0)=\left(\begin{array}{l}
1 \\
0
\end{array}\right) \mathrm{e}^{\mathrm{i} p_{+} x}+b\left(\begin{array}{l}
1 \\
0
\end{array}\right) \mathrm{e}^{-\mathrm{i} p_{+} x} \\
& +a\left(\begin{array}{l}
0 \\
1
\end{array}\right) \mathrm{e}^{\mathrm{i} p_{-} x} .
\end{aligned}
$$

The momenta $p_{ \pm}$are close to the Fermi momentum $p_{F}$ and are fixed by the energy, $E$. The amplitudes for electron reflection, $b$, and Andreev hole reflection, $a$, allow us to obtain the differential conductance as $g_{s}=1+|a|^{2}-|b|^{2}$, whereas the normal state conductance is just $g_{n}=1-|b|^{2}$. We shall consider here only $k_{y}=0$ or $\pi$. The transmitted waves into the MBS are superpositions of wavevectors $\boldsymbol{k}^{ \pm}, \boldsymbol{q}^{ \pm}$from two FS pockets (see Fig. 1) and the wave function for $x \geq 0$ is $\exp \left(\mathrm{i} k_{y} y\right) \psi_{S}$ where

$$
\begin{aligned}
& \psi_{S}(x \geq 0)=C \phi_{\boldsymbol{k}^{+}} \mathrm{e}^{\mathrm{i} k^{+} x}+\phi_{\boldsymbol{k}^{-}} D \mathrm{e}^{-\mathrm{i} k^{-} x} \\
& +E \phi_{\boldsymbol{q}^{+}} \mathrm{e}^{i q^{+} x}+F \phi_{\boldsymbol{q}^{-}} \mathrm{e}^{-i q^{-} x}
\end{aligned}
$$

where each $\phi_{\boldsymbol{k}}$ denotes a four-dimensional column eigenvector of the BdG matrix, $\widehat{H}_{S}(\boldsymbol{k})$. The $x$-components of the momenta, $k^{ \pm}, q^{ \pm}$, are chosen so that the group velocity is positive for energy $E$ above the gap. For subgap energies, the momenta have a positive imaginary part.

In QWT the matching conditions for wave functions (3) and (4) at $x=0$ are written as $[5,6]$ :

$$
\begin{aligned}
& \psi_{N}(0) \otimes\left(\begin{array}{l}
1 \\
1
\end{array}\right)=\psi_{S}(0), \\
& \partial_{k_{x}} \widehat{H}_{N} \psi_{N}(0)=\left(\begin{array}{llll}
1 & 1 & 0 & 0 \\
0 & 0 & 1 & 1
\end{array}\right) \cdot \partial_{k_{x}} \widehat{H}_{S} \psi_{S}(x=0) .
\end{aligned}
$$

Here, $H_{N}$ denotes a BdG Hamiltonian matrix for the normal metal. Interface disorder can be accounted for [5] by making the replacement: $1-b \rightarrow 1-b-2 i Z(1+$ b) $p_{F} / p_{+}$and $a \rightarrow a\left(1-2 i Z p_{F} / p_{+}\right)$in the right-hand side of Eq. (6), and where $Z$ denotes the BTK parameter [7].

\section{Results}

According to QWT [5], the condition for the existence of ABS is obtained from the $4 \times 4$ matrix $\Lambda$ composed of the four column vectors $\phi_{\boldsymbol{k}^{+}}, \phi_{\boldsymbol{k}^{-}}, \phi_{\boldsymbol{q}^{+}}, \phi_{\boldsymbol{q}^{-}}$, in Eq. (4). The condition then reads

$\operatorname{det} \Lambda=0$.

We checked that condition (7) does not hold for transverse momentum $k_{y}=0$ either in the topological $\left(t_{2}=\right.$ $-0.08)$ or in the non topological case $\left(t_{2}=+0.08\right)$. For transverse momenta $k_{y}=\pi$, Eq. (7) is verified only in the topological case. This means that the quantum interference effects from the two FS pockets effectively anihilate the two ABS's that would be predicted by single pocket BTK theory.

One might be tempted to read condition (7) as the requirement that the linear system in Eq. (5) be homogeneous and the wave function $\psi_{S}$ be made to vanish [11] at the N/S boundary. This would be incorrect, however, 
as it would imply the conductance $g_{s}$ to vanish. On the contrary, the conductance $g_{s}$ is finite and independent of the disorder parameter $Z$ at the energy value where Eq. (7) is obeyed $[5,7,10]$.

For the non-topological case, the differential conductance at normal incidence, $k_{y}=0$, is shown in Fig. 4 (left). It is seen that the quantum interference supresses quasi-particle transmission, $g_{s}$, as $E \rightarrow 0$ and the effect is even more pronounced as disorder increases. A similar result is obtained at $k_{y}=\pi$. Features such as peaks and dips are visible when the energy $E$ crosses the superconducting gaps on the Fermi pockets. For the topological case, the differential conductance at normal incidence, $k_{y}=0$, is shown in Fig. 5. A similar destructive interference is observed at low energy.

For transverse momentum $k_{y}=\pi$, the ABS (MF) leaves its imprint on the conductance, as Fig. 6 shows. The differential conductance attains the maximum value $g_{s}=2$ at $E=0$, independent of $Z$.

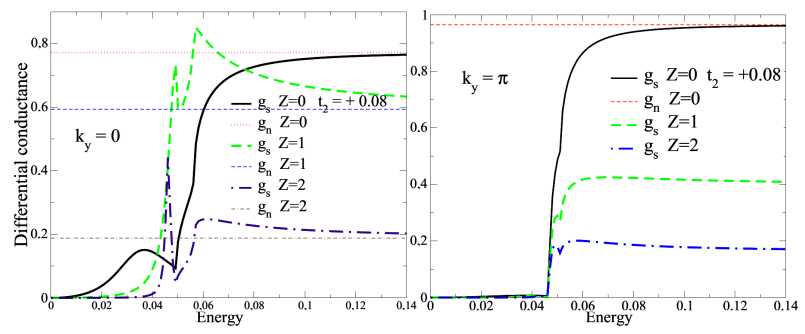

Fig. 4. Differential conductance at normal incidence (left) and for $k_{y}=\pi$ (right). $t_{2}=0.08$. Dashed line: normal state differential conductance.

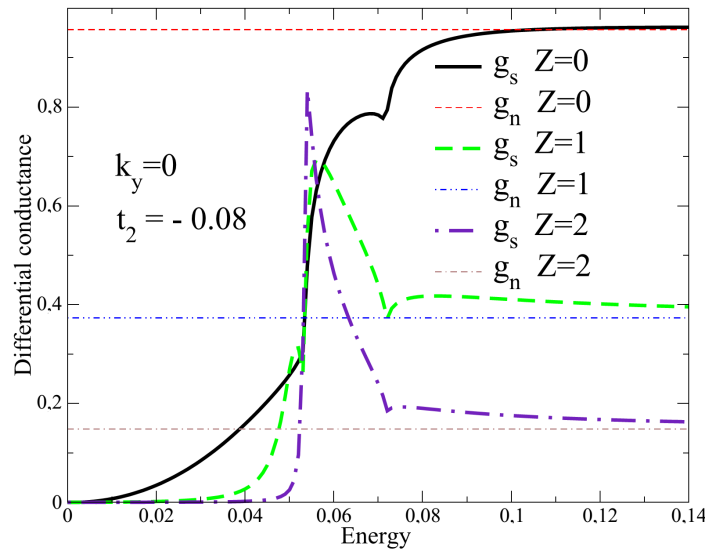

Fig. 5. Differential conductance at normal incidence, for $t_{2}=-0.08$. Dashed line: normal state differential conductance.

\section{Conclusions}

When the number of Fermi pockets is odd, the MF is in the single pocket that is traversed by the quasi-particles. The other pair of pockets interfere destructively, reconciling the ABS number with the topological index. In addition to the destruction of the ABS's, the waveguide

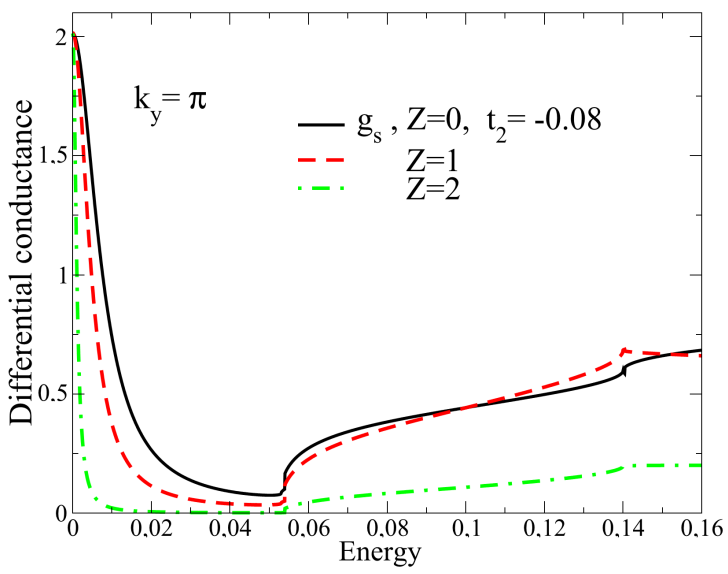

Fig. 6. Differential conductance for $k_{y}=\pi$ and $t_{2}=$ -0.08 .

interference effects also produce a vanishing conductance at $E=0$, when a pair of FS pockets is traversed by the quasi-particles.

\section{Acknowledgments}

We would like to thank financial support from Fundação para a Ciência e Tecnologia (Project EXPL/FIS-NAN/1728/2013 and Grant No. PESTOE/FIS/UI0091/2011).

\section{References}

[1] M.Z. Hasan, C.L. Kane, Rev. Mod. Phys. 82, 3045 (2010).

[2] X.-L. Qi, S.-C Zhang, Rev. Mod. Phys. 83, 1057 (2011).

[3] J. Alicea, Rep. Prog. Phys. 75, 076501 (2012).

[4] M.A.N. Araújo, E.V. Castro, P.D. Sacramento, Phys. Rev. B 87, 085109 (2013).

[5] M.A.N. Araújo, P.D. Sacramento, Phys. Rev. B 79, 174529 (2009).

[6] M.A.N. Araújo, P.D. Sacramento, J. Phys. Conf. Series 200, 012008 (2010).

[7] G.E. Blonder, M. Tinkham, T.M. Klapwijk, Phys. Rev B 25, 4515 (1982).

[8] A.C. Silva, M.A.N. Araújo, P.D. Sacramento, Europhys. Lett. 11037008 (2015).

[9] Y. Hatsugai, T. Fukui, H. Aoki, Phys. Rev. B 74, 205414 (2006).

[10] S. Kashiwaya, Y. Tanaka, M. Koyanagi, K. Kajimura, Phys. Rev B 53, 2667 (1996).

[11] A. Yamakage, K. Yada, M. Sato, Y. Tanaka, Phys. Rev B 85, 180509(R) (2012). 